\title{
calcul des pieux sous sollicitations cycliques par la méthode des éléments finis
}

\author{
M. BOULON \\ Maître-assistant à l'université scientifique et médicale de Grenoble
}

A. PUECH

Ingénieur de recherche à l'institut français du pétrole actuellement ingénieur en chef Société Geodia

\section{INTRODUCTION}

Les auteurs présentent une méthode de simulation numérique du comportement des pieux soumis à des chargements cycliques axiaux du type de ceux que l'on rencontre sur les fondations de certaines structures utilisées dans l'exploitation pétrolière en mer (platesformes treillis - plates-formes à ancrages tendus).

L'Institut français du pétrole a entrepris en 1978, pour le compte de l'Association de Recherche en Géotechnique Marine (A.R.G.E.M.A.), un vaste programme de recherche sur la tenue des pieux à l'arrachement. Sur le plan expérimental, ce programme s'est concrétisé par la mise en place (battage) et l'essai de deux pieux fortement instrumentés sur les sites de Plancoet et Cran. Les résultats de nombreuses séries d'essais de chargement statique et cyclique effectués sur ces deux pieux ont été partiellement publiés (Puech et al. [22], [23], [24]).

L'Institut de mécanique de Grenoble est engagé depuis de nombreuses années dans un programme d'étude en laboratoire des pieux sous charge axiale par simulation physique (Foray [15], Foray et Puech [16]) et par simulation numérique (Boulon et al. [4], [5], [6], [7],
[8]). Dans ce dernier domaine, le code de calcul Cyclope, application aux conditions de sollicitation des structures marines de travaux plus généraux menés à l'I.M.G., a été mis au point grâce à l'aide financière de l'I.F.P.

Le principe de simulation mis en œeuvre repose sur la modélisation des phénomènes physiques majeurs intervenant dans le comportement de l'ouvrage calculé et sur l'utilisation exclusive de parametres mesurables (contrairement aux méthodes de calcul a postériori): la méthode des éléments finis en déplacements est appliquée au sol et au pieu, tandis que les interactions de contact, incluant des déplacements relatifs sol-pieu, sont régies par la loi de Coulomb. La simulation numérique est la seule méthode permettant une synthèse simple de l'ensemble des propriétés mesurées, tant pour le sol et le pieu que pour l'interface. Toutefois, les calculs présentés ci-après sont quasi statiques et ne font pas intervenir de couplage solfluide interstitiel; ils s'appliquent donc à des sollicitations lentes (périodes de quelques secondes).

Le procédé de calcul retenu pour la simulation de l'effet des cycles a été suggéré par des considérations pratiques: il ne semble pas raisonnable, à l'heure actuelle, de calculer incrémentalement un ouvrage tel 
qu'un pieu soumis à un très grand nombre de cycles, et ceci pour deux raisons:

- D'une part, la connaissance des lois rhéologiques des sols, notamment sous sollicitation cyclique, est insuffisante, et conduit, quelle que soit la loi, à une dérive et à une instabilité importante sur chemin homogène non rectiligne, ceci étant déjà sensible à moins de 10 cycles (cf. conclusions du Workshop de Grenoble [11])

- D'autre part, si l'on admet qu'un cycle doit être décrit incrémentalement par 20 incréments, une sollicitation comportant 1000 cycles (ce qui est relativement faible dans le contexte marin) nécessitera 20000 incréments de conditions aux limites, sans préjuger du nombre d'incréments nécessaires à la simulation de l'installation du pieu. Dans l'état actuel des possibilités,

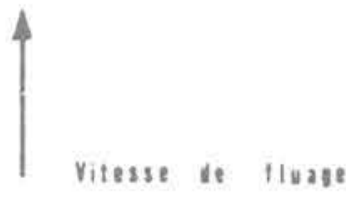

zxiale ( 3 , minute )
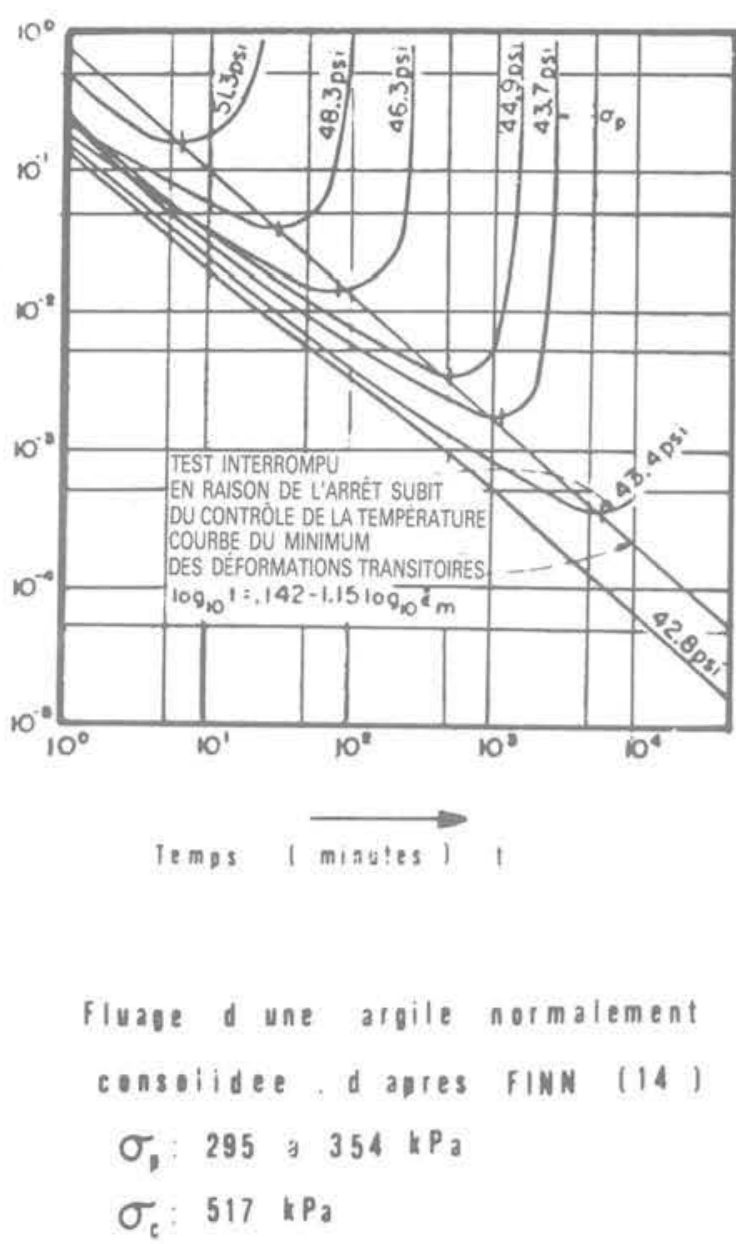

(a) le traitement d'un problème aux limites avec 20000 incréments reste peu réaliste, même avec un ordinateur vectorisé.

On s'est donc attaché à imaginer une méthode conduisant à un volume de calcul compatible avec les exigences courantes.

Différents procédés de «condensation » de l'effet d'un grand nombre de cycles ont été imaginés par les auteurs traitant de problèmes cycliques. Matlock et Foo [20] utilisent avec la méthode des coefficients de raideur, un modèle de sol sous cisaillement direct à hystérésis et à résistance mécanique décroissante avec le nombre de cycles ( hhystérétic and degrading soil model »). Poulos [21], travaillant sur la base des formulations de Mindlin prend en compte l'effet des cycles par l'intermédiaire de facteurs globaux de dégradation<smiles>[C+]=C</smiles>
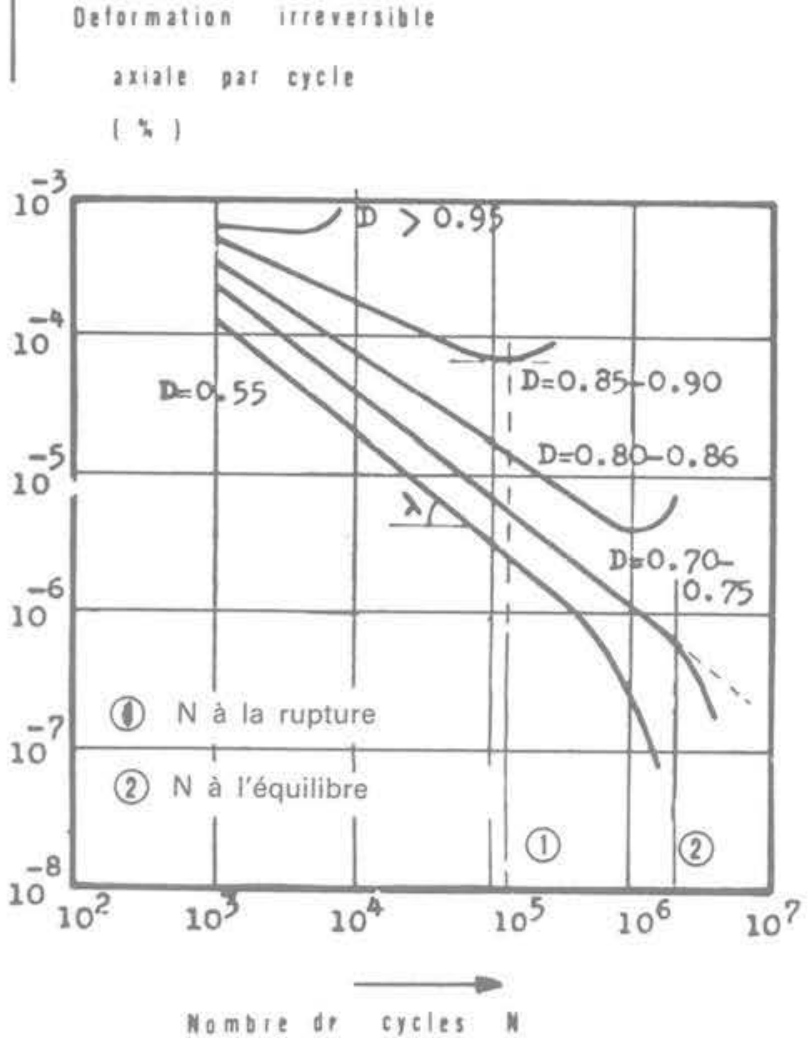

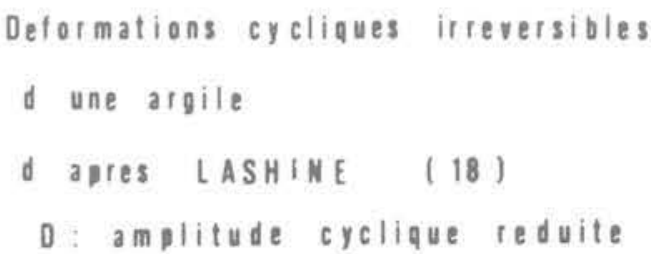

(b)

Fig. 1. - Analogie fluage / déformations cycliques irréversibles. 
( « cyclic degradation effects $»$ ). Cathie [10] propose également un modèle de sol en contraintes totales avec effet progressif des cycles regroupés. Enfin, Bonin et al. [3] présentent un calcul de structure offshore à embase poids, par la méthode des éléments finis, dans lequel l'effet de nombreux cycles est simulé par une assimilation au fluage des solides visqueux.

On présente dans une première partie les bases physiques du modèle rhéologique de sol sous sollicitation cyclique partiellement utilisé dans notre simulation numérique, puis le modèle numérque lui-même, et enfin des résultats de calculs appliqués au pieu de Plancoet.

\section{BASES PHYSIQUES DU MODĖLE DE SOL SOUS SOLLICITATION CYCLIQUE}

Il existe une grande analogie formelle entre le fluage et laccumulation des déformations inéversibles cycliques, qu'on peut apprécier à partir de la figure 1 où sont représentées pour deux argiles de propriétés mécaniques voisines les courbes traduisant la vitesse axiale de fluage en fonction du temps avec comme paramètre le déviateur axial de contrainte (d'après Finn et al. [14]) et parallèlement les courbes représentant la déformation irréversible par cycle en fonction du nombre de cycles, avec comme paramètre l'amplitude cyclique réduite (d'après Lashine [18]). On est donc tenté de poursuivre l'analogie en assimilant le nombre de cycles à un pseudo-temps, le déviateur cyclique moyen a un pseudo-déviateur de fluage, l'amplitude du déviateur cyclique intervenant comme paramètre secondaire, ceci étant valable sur chemin biaxial de révolution. Bien

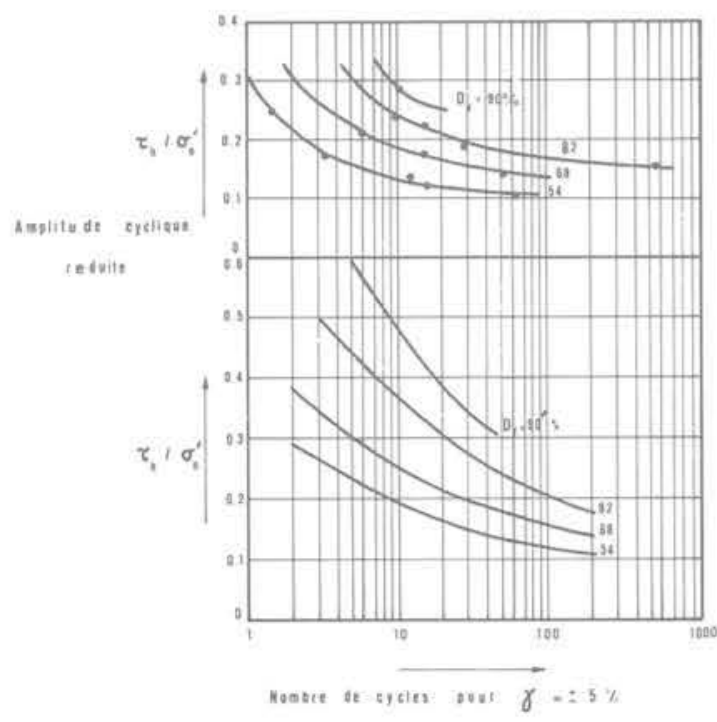

(a) SABLE DE MONTEREY $N^{\prime} D$ - INFLUENCE DE 0 D. APRES SEEO [22] entendu les vitesses de fluage proprement dit et de pseudo-fluage cyclique sont a priori sans relation.

Si la sollicitation est composée de cycles de caractéristiques variées, en amplitude notamment, les concepts précédents doivent être complétés par la notion de cycles équivalents. En d'autres termes, on constate que les effets d'un petit nombre de grands cycles et d'un grand nombre de petits cycles sont voisins, aussi bien sur sable (cf. fig. 2a, d'après Seed [25]) que sur argile (cf. fig. 2b, d'après Andersen [1]). Le paramètre densité relative ( $\mathrm{Dr}$ ) intervient évidemment pour les sables, au même titre que le degré de surconsolidation (O.C.R.) pour les argiles. Van Eekelen [13] a posé les principes de l'approche par cycles équivalents à partir de la loi de Miner.

Les autres phénomènes liés aux sollicitations cycliques sont nombreux. Les plus importants du point de vue du comportement du sol sont les variations de volume sous cycles, le durcissement ou le radoucissement cyclique. La figure $3 a$ précise ces notions: sur chemin biaxial de révolution, et à densité relative élevée, le sable d'Hostun est dilatant sur chemin monotone, mais contractant sur chemin cyclique (d'après Foray [17]).

Les variations de volume cycliques sont quantifiables à partir du concept d'état caractéristique (cf. Luong [19]). L'examen des figures $3 \mathrm{a}$ et $3 \mathrm{~b}$ permet de constater un durcissement cyclique pour les cycles à $(50 \pm 25) \%$ du déviateur de rupture (le module sécant cyclique croît avec le nombre de cycles), et par contre un radoucissement cyclique pour des cycles à (75 \pm 25) \% du déviateur de rupture.

L'idée de base dans le modèle actuel de sol sous sollicitation cyclique a donc été d'assimiler les modifications dues à ces sollicitations à un pseudo-fluage
Amolituet sycligue reasits

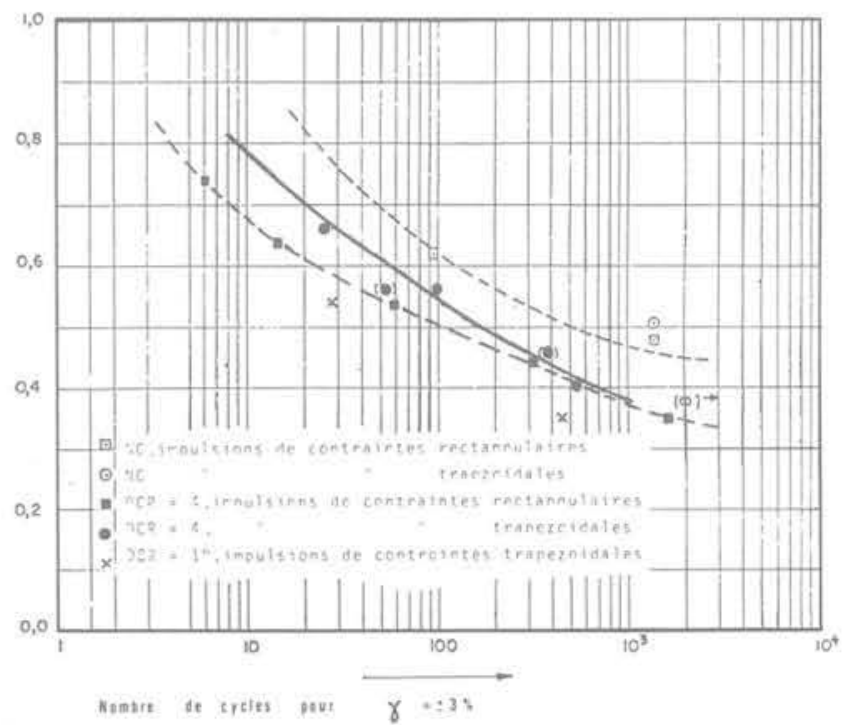

(b) ARGILE OE DRAMMEN - INFLUENCE DE O.C.R.

D APRES ANDERSEN [ 1 ]

Fig. 2. - Cisaillement cyclique non drainé - notion de cycles équivalents. 

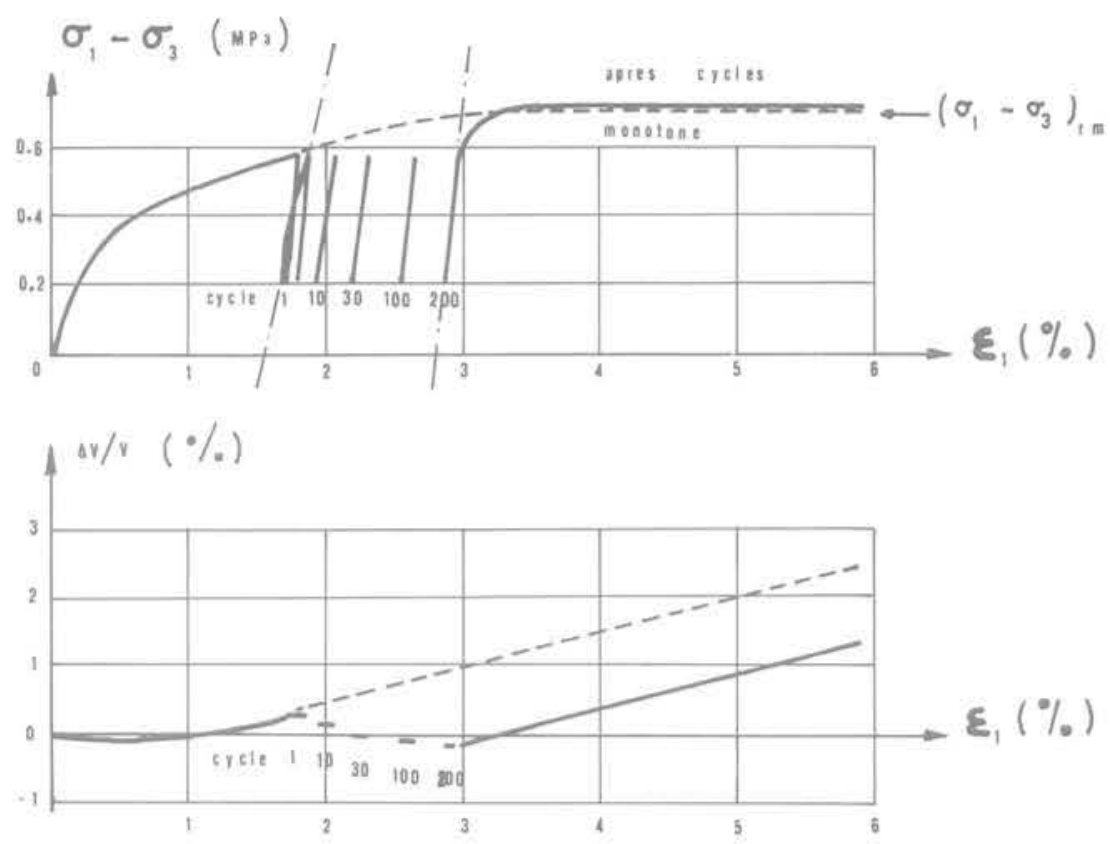

(a) deviateut cyclique $(50 \%: 25 \%) \cdot\left(\sigma_{1}-\sigma_{3}\right)$ im . dilatance monotone - conteactance at durcissement cycliques

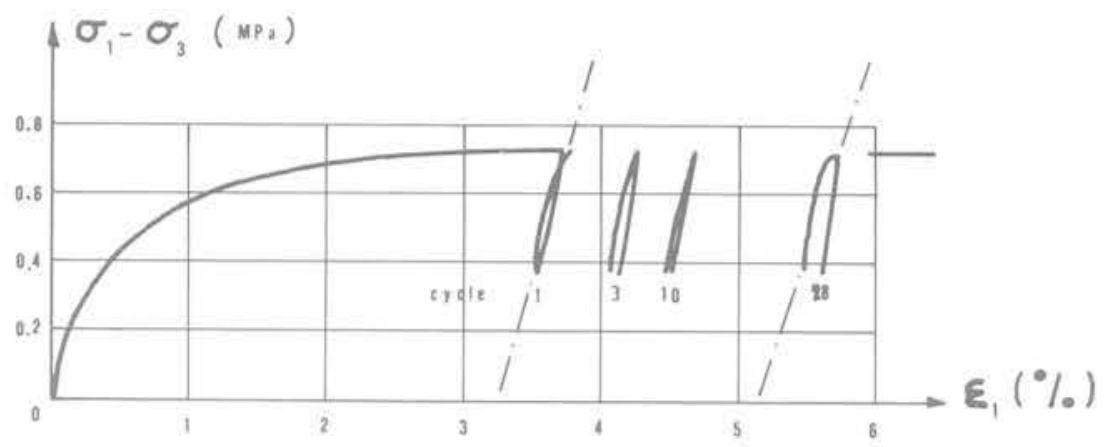

(b) deviateur cyclique
- tadoucissement

Fig. 3. - Chemins triaxiaux cycliques drainés - sable d'Hostun $\sigma_{3}^{\prime}: 0,2 \mathrm{MPa}-\gamma_{d}: 1,8110^{4} \mathrm{~N} / \mathrm{m}^{3} \mathrm{D}_{\mathrm{r}} \not 11$ Comportements typiques d'après Foray [17].

cyclique restant à préciser; on notera toutefois que parmi les phénomènes mentionnés ci-dessus, certains sont à quantifier en tant que tels (déformation axiale irréversible, variations de volume) tandis que d'autres peuvent être considérés comme conséquence des premiers (durcissement ou radoucissement cycliques).

\section{LE MODÈLE NUMÉRIQUE}

Le modèle numérique est la schématisation du système sol, pieu, interface sol-pieu, muni des diverses lois de comportement nécessaires pour représenter l'ensemble. On doit bien entendu distinguer le problème continu du problème approché discrétisé.

\subsection{Le problème continu}

La géométrie est axisymétrique, et les hypothèses classiques de la mécanique des milieux continus sont reprises, à savoir: petites déformations, petites rotations, petits déplacements et petits déplacements relatifs (les déplacements relatifs sont nécessaires à l'interface).

La mise en équation est classique également, en déplacements, à partir du principe du travail virtuel en termes de vitesses de déplacement, de déformation et de contrainte. Ce principe du travail virtuel est explicité pour un solide unique, soumis à des conditions aux limites en déplacement (sur $\Gamma_{\mathrm{u}}$ ) et en contraintes (sur $\Gamma_{o}$ ), fig, $4 \mathrm{a} ; \delta \dot{u}_{i}, \delta \dot{\varepsilon}_{i j}, \dot{\sigma}_{i j}, f_{1}$ sont respectivement les champs de vitesse de déplacement virtuel sur $\Omega$, de vitesse de déformation virtuelle sur $\Omega$ correspondant à 
$\delta \dot{u}_{\mathrm{i}}$, de vitesse de contrainte réelle sur $\Omega$ et de vitesse de forces de volumes réelles sur $\Omega$. Cette écriture est équivalente à l'ensemble (équations d'équilibre, conditions aux limites) classique en mécanique des milieux continus, mais plus adaptée au type de résolution choisí.

Le problème est sensiblement compliqué ici par la présence de deux solides en contact sur un interface $\Gamma_{s}$ constitué par le fât et la pointe du pieu. Le principe du travail virtuel évoqué ci-dessus doit être modifié pour tenir compte des déplacements relatifs éventuels selon cet interface; il prend la forme indiquée fig. $4 \mathrm{~b}$ (cf. Boulon [9] pour plus de détails). Dans cette expression, $\Gamma_{\mathrm{sc}}, \Gamma_{\mathrm{sg}}$ et $\Gamma_{\mathrm{sr}}$ sont respectivement les parties de l'interface oû sol et pieu sont collés $\left(\Gamma_{\mathrm{sc}}\right)$, en état de glissernent relatif $\left(\Gamma_{\mathrm{sq}}\right)$ et décollés $\left(\Gamma_{\mathrm{st}}\right)$, ceci en fonction d'un critère de contact de type Coulomb, par exemple $\left(|\tau| \leqslant \sigma_{n}, \operatorname{tg} \delta+a\right) ;(\delta:$ angle de frottement d'interface, a adhérence d'interface). L'écriture du principe des travaux virtuels fait ici apparaître $\delta \dot{g}_{\mathrm{ti}}$ champ de vitesse de déplacement relatif virtuel sur l'interface, ainsi que $\Delta \Gamma_{\mathrm{sg}}$, supplément d'extension de $\Gamma_{\text {sg }}$ entre les temps $t$ et $t+d t$.

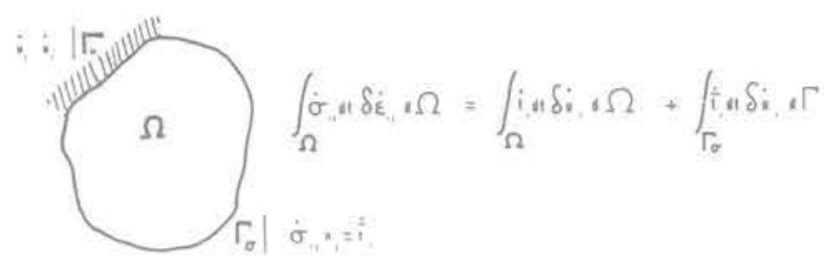

(4) Wh soitor

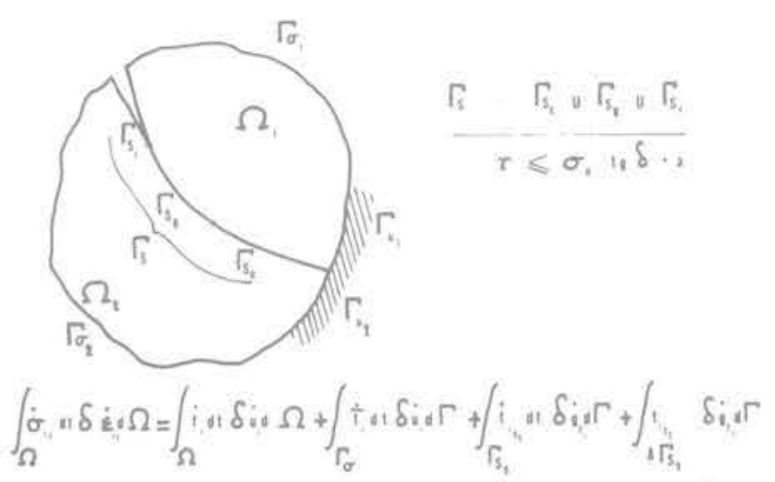

(в) Deuz solides initiatement en contaet sur $\Gamma_{\text {s }}$

\section{Fig. 4. - Principes des puissances virtuelles en termes de vitesses.}

On suppose le pieu élastique linéaire; toutefois, on néglige ses déformations transversales en lui affectant un coefficient de Poisson nul. En ce qui concerne le sol, on fait appel à deux lois rhéologiques. L'une sur chemin monotone ([4]). C'est une relation fonctionnelle de chemin à chemin entre l'espace des déformations et celui des contraintes faisant intervenir l'indice des vides initial, et ne faisant pas intervenir le temps.

$$
\mathscr{F}\left(\sigma_{i j}, \varepsilon_{i j}, e_{o}, \ldots\right)=0
$$

avec $\sigma_{i j}$ : histoire des composantes du tenseur contraintes

$\varepsilon_{\mathrm{jj}}$ : histoire des composantes du tenseur déformations.

L'autre loi rhéologique s'applique aux chemins cycliques à niveaux moyens de contraintes et amplitude cyclique en contraintes constants. C'est une loi de pseudo-fluage cyclique qui constitue la partie pseudovisqueuse de la loi précédente (le nombre de cycles tient lieu du temps dans une loi réellement visqueuse). L'hypothèse majeure relative à ce type de comportement est que les propriétés de pseudo-fluage cyclique du sol ne dépendent que du chemin cyclique moyen, de l'amplitude des écarts au chemin cyclique moyen, et de l'indice des vides initial du sol.

\subsection{Le problème discrêtisé}

La discrétisation porte sur l'espace, les conditions aux limites, et les propriétés mécaniques.

La discrétisation de l'espace est celle de la méthode des éléments finis, qui permet une mise en équation élément par élément.

La discrétisation des conditions aux limites fait qu'à un incrément de conditions aux limites correspond un incrément du champ de déplacement, du champ de déformation et du champ de contraintes. La forme discrétisée de l'équation (2) est l'équation (4) (cf. fig. $5 \mathrm{a}$ ) où

$\Sigma_{e}$ a le sens d'une somme algébrique sur les éléments (we élément courant);

$\left(\Delta \sigma_{i j}\right)^{q}$ est l'incrément du champ de contraintes réelles correspondant à l'incrément q de conditions aux limites;

$\left(\Delta \mathrm{f}_{1}\right)^{\mathrm{q}}$ est l'incrément du champ de forces de volume correspondant à l'incrément $q$ de conditions aux limites.

Les champs virtuels $\delta \dot{u}_{i}, \delta \dot{\varepsilon}_{i j}$ et $\delta \dot{g}_{t i}$ sont également ceux qui correspondent à l'incrément $\mathrm{q}$ de conditions aux limites.

Sur chemin monotone, la discrétisation des propriétés mécaniques a conduit à une loi rhéologique incrémentale orthotrope octolinéaire L 1 (généralisation tridimensionnelle du concept monodimensionnel de charge décharge) (cf. fig. 5b) décrite par Boulon et al. [4], et largement perfectionnée depuis son origine (cf. Darve [12]). Le choix de la détermination incrémentale est fait avec un incrément de retard. L'identification de cette loi est réalisée sur chemins biaxiaux de révolution en compression et en extension.

Sur chemin cyclique, le calcul du niveau cyclique moyen réduit et de l'amplitude cyclique réduite selon chaque direction d'orthotropie donnent accès aux déformations axiales et aux déformations latérales selon chacune de ces directions (cf. fig. $5 \mathrm{c}$ ). Le calage de cette loi de pseudo-fluage cyclique L 2 nécessite des essais biaxiaux de révolution drainés à divers niveaux cycliques moyens et à diverses amplitudes cycliques. 


$$
\begin{aligned}
& \sum_{i} \int_{\omega_{\varepsilon}}\left(\Delta \sigma_{i,}\right)^{i} \delta \dot{\varepsilon}_{i,} d \Omega=\sum_{i} \int_{\omega_{i}}(\Delta f)^{i} \delta_{i j} d \Omega \\
& \text { inerement a tes concitions ave limites }
\end{aligned}
$$

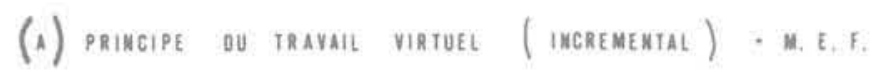
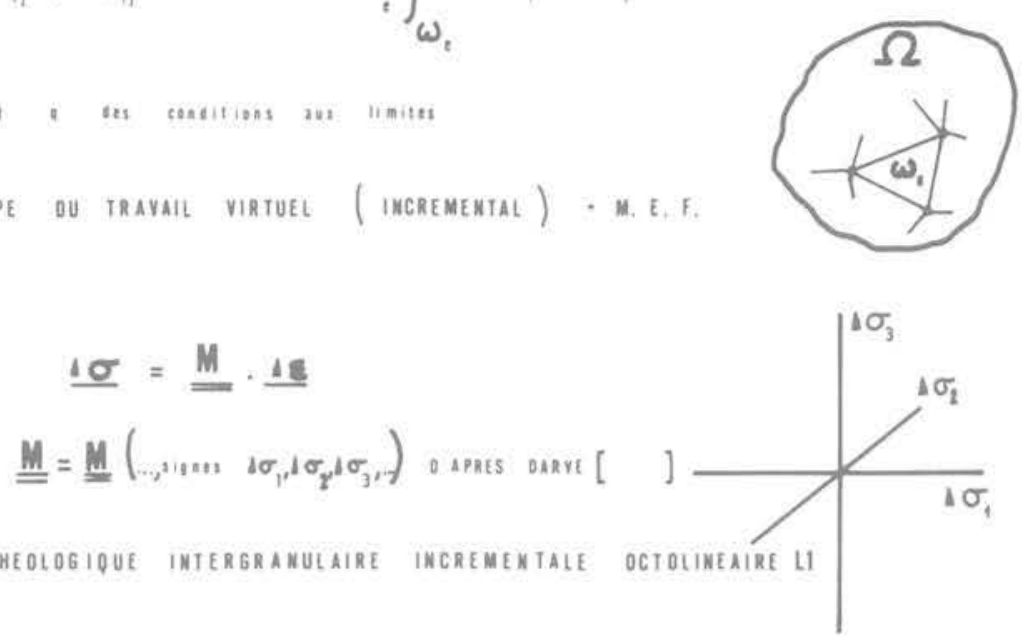

(a) Loi rheologique intergranulatre incrementale octolimeaire LI

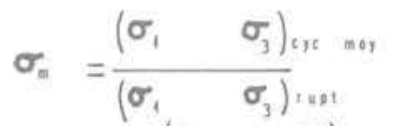

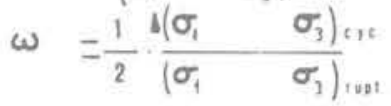

$$
\begin{aligned}
& \varepsilon_{1}[n]=\omega^{m} \cdot\left(\frac{1 \cdot \sigma_{m}}{1-\sigma_{m}}\right)^{n} \cdot \frac{n}{1+\left[1+\left(\omega+\sigma_{m}\right)\right]^{n} \cdot n} \\
& \therefore=k_{2}\left(\sigma_{3}\right)^{2} \\
& \text { - }=\mathrm{k}_{\mathrm{g}}\left(\sigma_{3}\right)^{\prime}
\end{aligned}
$$

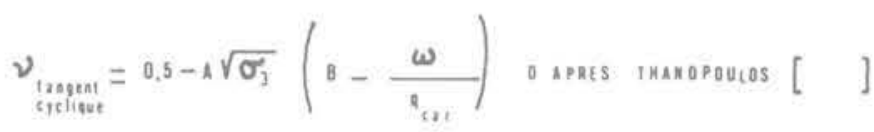

(c) Lol rheologique intergramulaire sur chemin cyclioue 12

Fig. 5. - Discrétisation (espace, conditions aux limites et propriétés mécaniques),

La mise en cuure de calculs cycliques nécessite quelques précisions complémentaires. Il s'agit d'un calcul en déplacements asservi en force, qui permet de conserver tout son sens à un incrément qui atteint le palier en force d'arrachement en tête du pieu. Le calcul des glissements relatifs est réalisé par la méthode des coefficients d'influence décrite par Boulon et al. ([6] et [7]). Les cycles composant la sollicitation sont regroupés par séries d'amplitude constante en termes de forces (ce qui est souvent le cas des sollicitations naturelles), le nombre de cycles de chaque série étant d'autant plus important que leur amplitude est faible. L'utilisation de la loi L 2 nécessite la connaissance en chaque point du sol des cycles en contraintes dus aux cycles en force en tête du pieu. C'est la loi L 1 qui permet de parvenir à ce résultat par un parcours incrémental complet du premier cycle de chaque série. Cette méthode est résumée à la figure 6 qui indique notamment que la loi $L 1$ intervient également dans les cycles «extrapolés» par pseudo-fluage cyclique (différence entre fluage homogène et hétérogène). Par ailleurs, il est clair que le durcissement et le radoucissement cyclique sont en partie inclus dans l'ensemble ( $\mathrm{L} 1, \mathrm{~L} 2$ ), puisqu'un des résultats du pseudo-fluage cyclique est une variation d'indice des vides du matériau, qui modifie évidemment ses propriétés mécaniques.

La simulation de l'installation du pieu à partir de l'état $\mathrm{Ko}$, notamment dans le cas du battage, est fort complexe, et correspond à l'évidence, d'une part à un serrage initial du milieu, d'autre part à des changements très mal connus des propriétés mécaniques de la couche limite située à proximité immédiate du pieu. Ne disposant pas encore de simulation adéquate (restant à développer), on a provisoirement traité cet aspect selon la méthode de Balaam et al. [2], mais cette approche doit être considérée comme insuffisante, d'autant que les paramètres pris en compte à ce stade ne sont pas directement mesurables. 


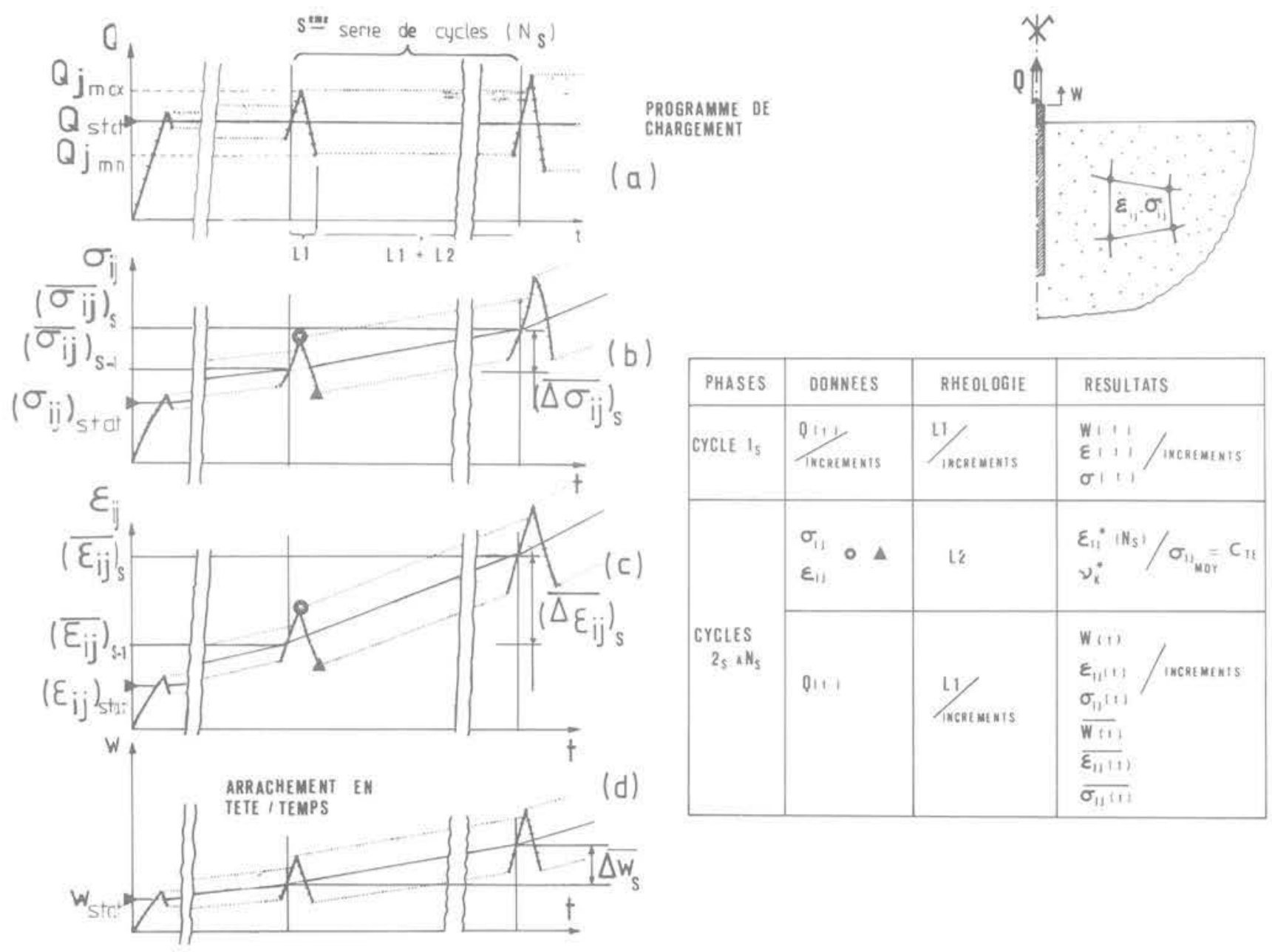

Fig. 6. - Modélisation du comportement par pseudo-fluage cyclique.

\section{SIMULATION NUMÉRIQUE DE L'ARRA- CHEMENT MONOTONE ET CYCLIQUE DU PIEU EXPÉRIMENTAL DE PLANCOET}

\subsection{Caractéristiques générales des calculs}

Le maillage utilisé, représenté à la figure 7, correspond à un pieu de $27 \mathrm{~cm}$ de diamètre et de $13 \mathrm{~m}$ de fiche. Le réseau nodal respecte les hétérogénéités du terrain constitué de trois couches nettement différenciées, à savoir, en partant de la surface, silt sableux, sable lâche et argile silteuse. Ces calculs ont été réalisés avec 96 nouds et 75 couples d'éléments à 3 nœeuds. Les caractéristiques mécaniques sont évaluées au centre de gravité de chaque couple d'éléments. La liaison pieusol en pointe à l'arrachement est simulée par une "galette» de résistance mécanique très faible lorsque l'adhérence limite pieu-sol est dépassée.

Le temps de calcul est voisin d'une minute C.P.U. par incrément sur l'ordinateur Honeywell-Bull 68 du Centre de calcul de Grenoble.

\subsection{Arrachement monotone}

L'essai statique S 2 de Plancoet a été choisi comme essai de référence. La figure 8 donne la relation effort-arrachement pour cet essai S 2, une simulation numérique $A$ de cet essai avec la loi $L 1$ seule, ne tenant pas compte de l'installation du pieu, et une simulation numérique $B$ avec la loi L 1 seule, utilisant des caractéristiques mécaniques améliorées de $50 \%$ en moyenne pour tenir compte de l'installation du pieu (cf. tableau I), puisque le comportement de chaque couche est contractant dans le domaine de contraintes latérales situé entre 0 et $200 \mathrm{kPa}$.

Les deux calculs ont êté réalisés avec 12 incréments. On remarquera que les deux simulations conduisent à un comportement du pieu plus souple que dans la réalité, ce quí est prévisible compte tenu de la remarque du $\$ 3.2$. relative à l'état initial. Le comportement du pieu au voisinage de la rupture est bien simulé dans le second cas, moyennement dans le premier cas. 
TABLEAU I. Caractéristiques mécaniques de base

\begin{tabular}{|c|c|c|c|c|c|c|c|c|}
\hline & \multirow{2}{*}{$\begin{array}{c}\mathrm{V} \\
\left(\mathrm{kN} / \mathrm{m}^{3}\right)\end{array}$} & & \multirow{2}{*}{$\begin{array}{l}\text { Ko } \\
\text { calculé }\end{array}$} & \multirow{2}{*}{$\begin{array}{c}\text { Module triaxial } \\
\text { de référence } \\
(\mathrm{kPA}) \\
\text { sous } \sigma_{3}=100 \mathrm{kPA}\end{array}$} & \multicolumn{2}{|c|}{$\begin{array}{l}\text { Caractéristiques } \\
\text { de volume à } \\
\text { la rupture }\end{array}$} & \multicolumn{2}{|c|}{$\begin{array}{c}\text { Caractéristiques } \\
\text { d'interface à } \\
\text { la rupture }\end{array}$} \\
\hline & & & & & $\operatorname{tg} \phi^{\prime}$ & $\mathrm{c}^{\prime}(\mathrm{kPa})$ & $\operatorname{tg} \delta$ & $\mathrm{a}(\mathrm{kPa})$ \\
\hline \multirow{3}{*}{$\begin{array}{l}\text { Simulation } \\
\text { sans } \\
\text { installation } \\
\text { pieu A }\end{array}$} & \multirow{3}{*}{18} & Silt................... & 0,59 & 15000 & 0,5 & 0 & 0,4 & 0 \\
\hline & & Sable lâche ..... & 0,59 & 30000 & 0,5 & 0 & 0,6 & 0 \\
\hline & & Argile silteuse & 0,43 & 13000 & 0,5 & 20 & 0,3 & 10 \\
\hline \multirow{3}{*}{$\begin{array}{l}\text { Simulation } \\
\text { avec } \\
\text { installation } \\
\text { pieu B }\end{array}$} & \multirow{3}{*}{18} & Silt................... & 0,59 & 30000 & 0,6 & 0 & 0,45 & 0 \\
\hline & & Sable lâche ...... & 0,59 & 45000 & 0,6 & 0 & 0,65 & 0 \\
\hline & & Argile silteuse & 0,43 & 19500 & 0,5 & 25 & 0,35 & 20 \\
\hline
\end{tabular}

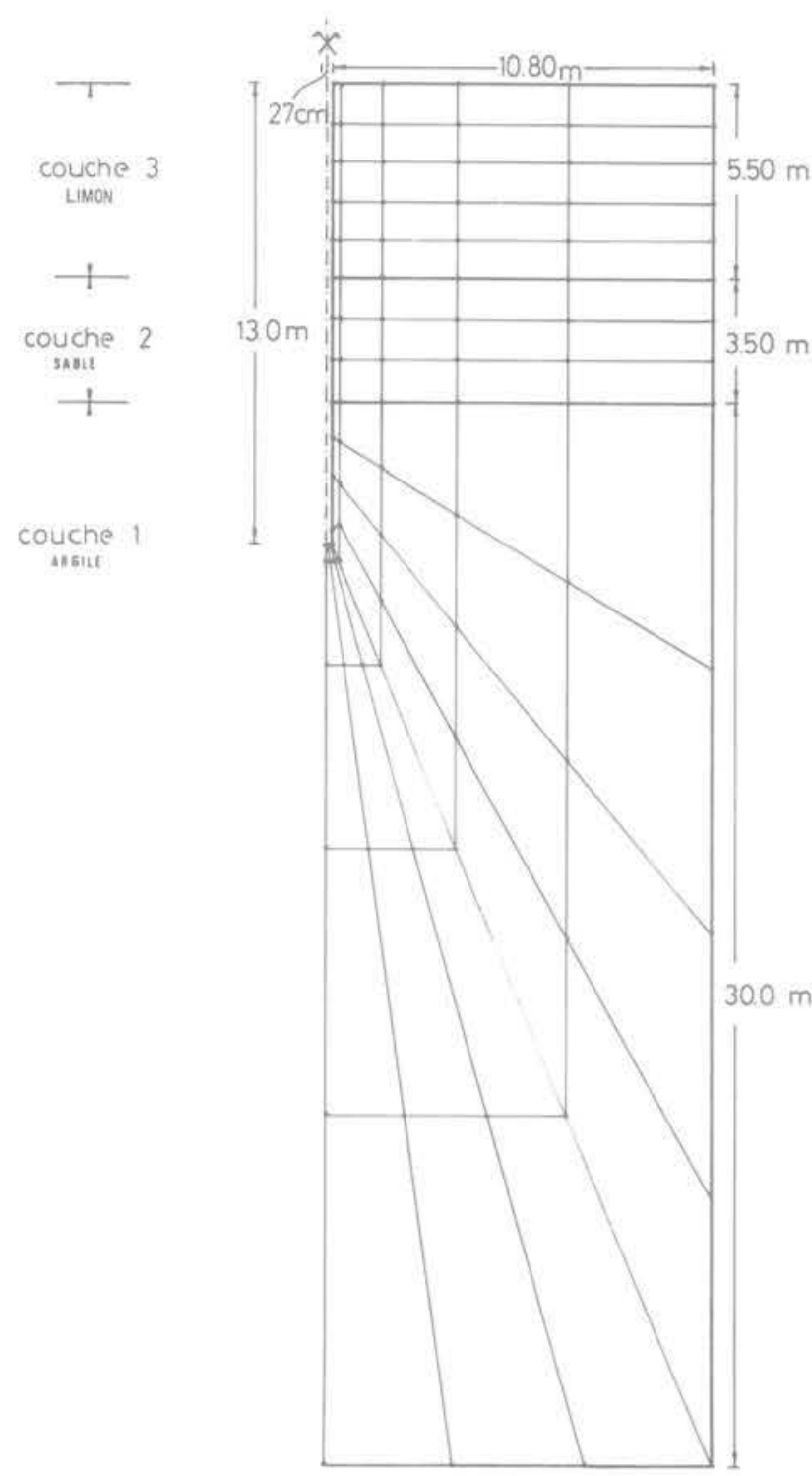

Fig. 7. - Maillage pieu expérimental Plancoet.

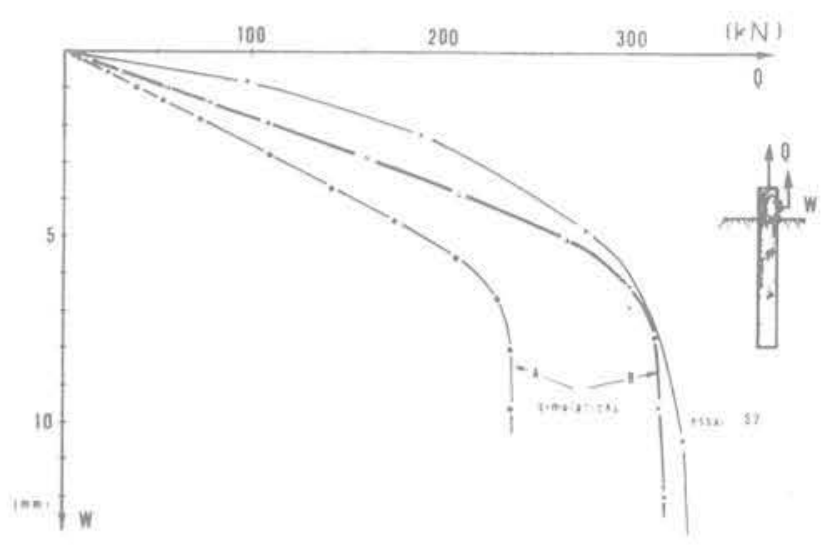

Fig, 8. - Arrachement statique lessai S2, simulations $A$ et $B$ )

Plancoet, courbe effort-arrachement.

La figure 9 permet de comparer la mobilisation des contraintes de cisaillement dans la couche de sable lâche, notamment vers $7,70 \mathrm{~m}$ de profondeur. On constate que la simulation $\mathrm{B}$ rend mieux compte tant du début de la courbe de mobilisation que du palier de contrainte de cisaillement.

Ce sont les caractéristiques mécaniques de la simulation B qui sont utilisées dans les calculs cycliques.

\subsection{Arrachement cyclique}

La simulation a porté sur deux essais:

- Un essai de longue durée à niveau cyclique moyen faible et à amplitude cyclique faible (essais C 12 ou C 21 de Plancoet).

- Un essai tempête, dont le niveau cyclique moyen est plus élevé et dont l'amplitude cyclique est croissante jusqu'à la rupture (essai T 2 de Plancoet). 


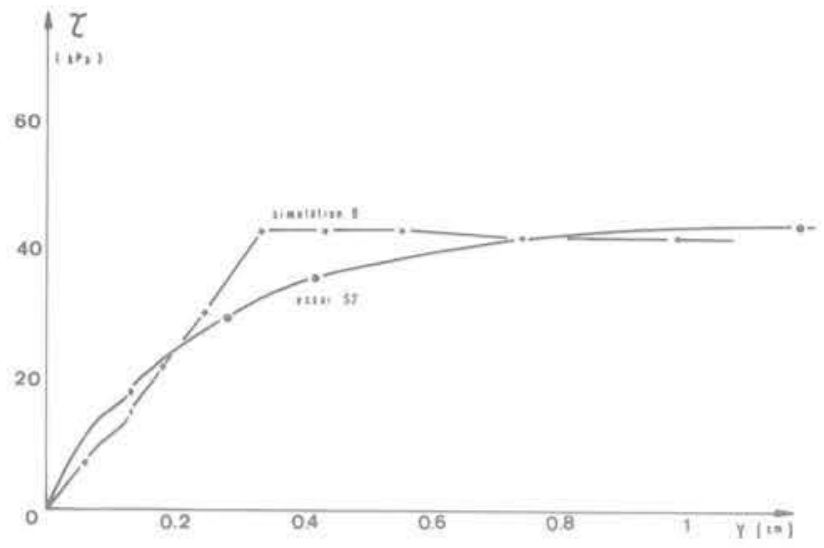

Fig. 9. - Arrachement statique Plancoet; mobilisation du frottement latéral

(couche intermédiaire; sable lâche. $Z=7,7 \mathrm{M}$ ).

\subsubsection{Simulation de l'essai cyclique} de longue durée (essai $C 12$ ) $-C-$

La charge conventionnelle de fluage mesurée était $\mathrm{Q}_{\mathrm{F}}$ $=270 \mathrm{kN}$; le chargement se caractérisant par 1000 cycles d'amplitude telle que:

$$
0,10 \leqslant \frac{Q}{Q_{F}} \leqslant 0,52 \text { ( } Q \text { charge en tête) }
$$

La simulation $\mathrm{C}$ a été réalisée en 21 incréments, dont 4 incréments de pseudo-fluage comme indiqué au $\S 3.2$.
La figure 10 montre que les déformations cycliques irréversibles sont concentrées sur les premiers cycles (simulation: sur les 16 premiers - expérience: sur les 200 premiers).

La figure 11 donne les modifications de contraintes de cisaillement agissant sur le fût, au cours des cycles, à l'intérieur de la couche de sable. On constate une augmentation de ces contraintes, après 1000 cycles, d'environ $10 \%$. On a constaté une augmentation légèrement supérieure expérimentalement.

La figure 12 ếvoque le transfert de charge sol-pieu simulé sous sollicitation statique (à comparer aux résultats de l'essai S 3) et après sollicitation cyclique (à comparer aux résultats de l'essai C 21 au bout de 1500 cycles). La tendance observée expérimentalement et par simulation est la même: chargement préférentiel dans la région basse du pieu, aux dépens de la région haute.

Enfin la figure 13 est consacrée à la comparaison des déplacements en tête en haut et bas de cycles (essai C 12). On constate que la simulation rend compte de l'amplitude, et de la variation du déplacement moyen entre le premier et le $1000^{e}$ cycle, mais n'est pas satisfaisante en ce qui concerne le déplacement moyen en tête avant le premier cycle. Ceci pose à nouveau le problème de l'état initial (après installation).

Dans cet ordre d'idées, nous avons représenté l'écart relatif des modules de frottement obtenu expérimenta-

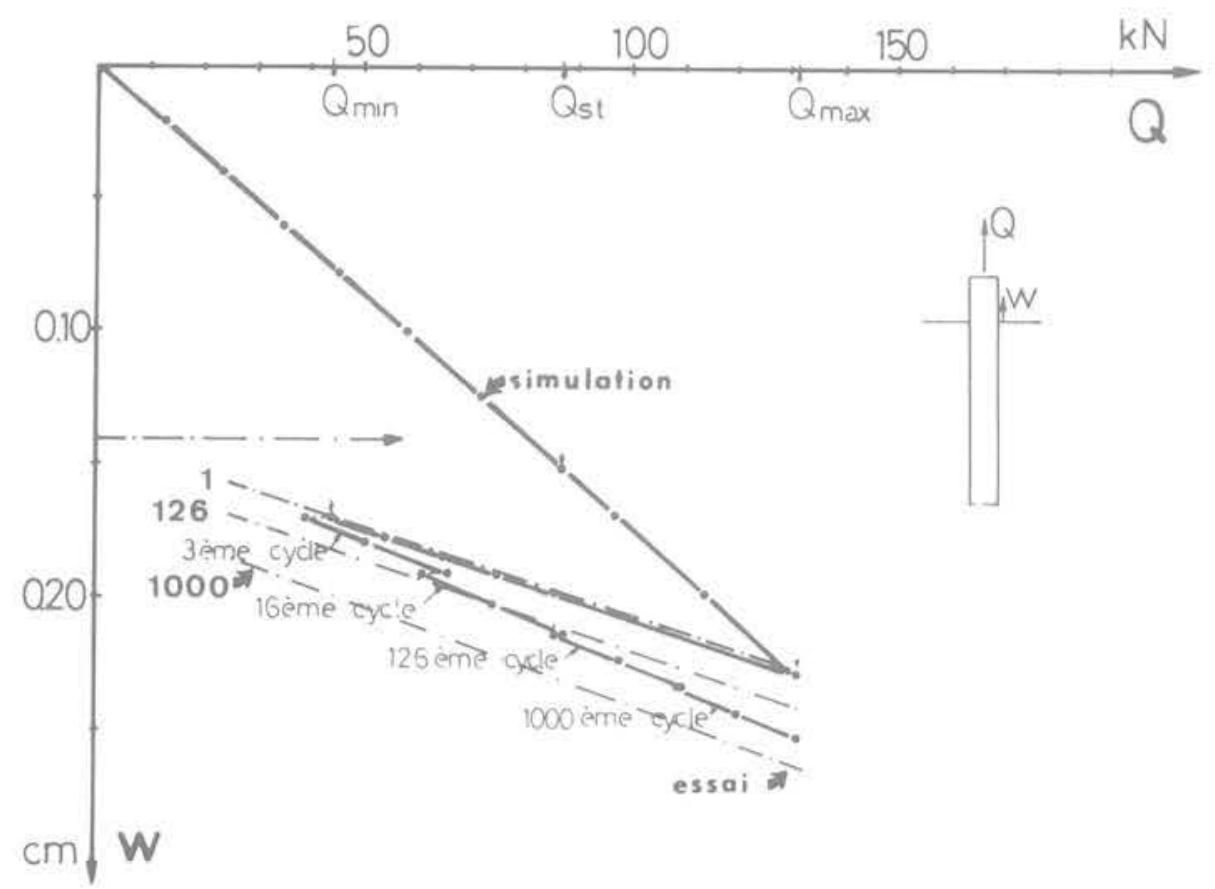

Fig. 10. - Plancoet. Essai cyclique C 12, courbe effort-arrachement

$$
\begin{array}{l|l}
1000 \text { crelis } & \begin{array}{l}
\text { ESSAI } 0.10 \leqslant \frac{0}{0} \leqslant 0.52 \\
\text { simulation } 0.17 \leqslant \frac{0}{0} \leqslant 0.53
\end{array}
\end{array}
$$




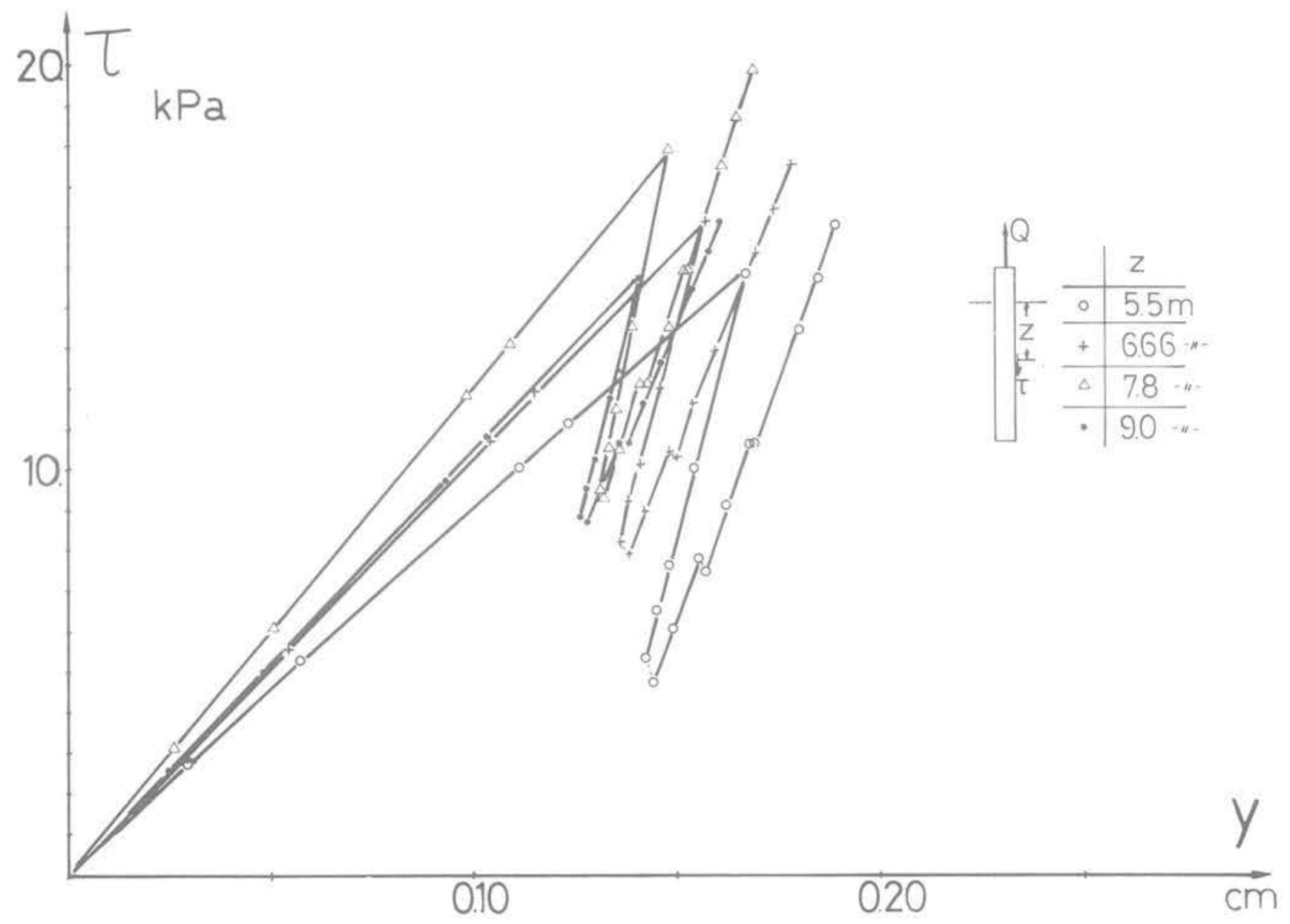

Fig. 11. - Plancoet essai C 12 simulation mobilisation du frottement latéral (couche intermédiaire - sable lâche).

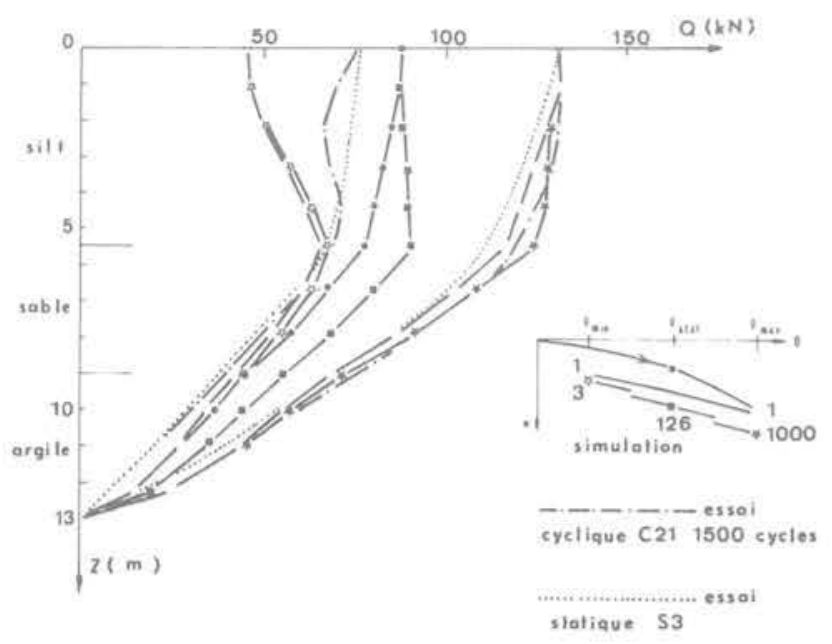

Fig. 12. - Plancoet transfert de charge sol-pieu après cycles.

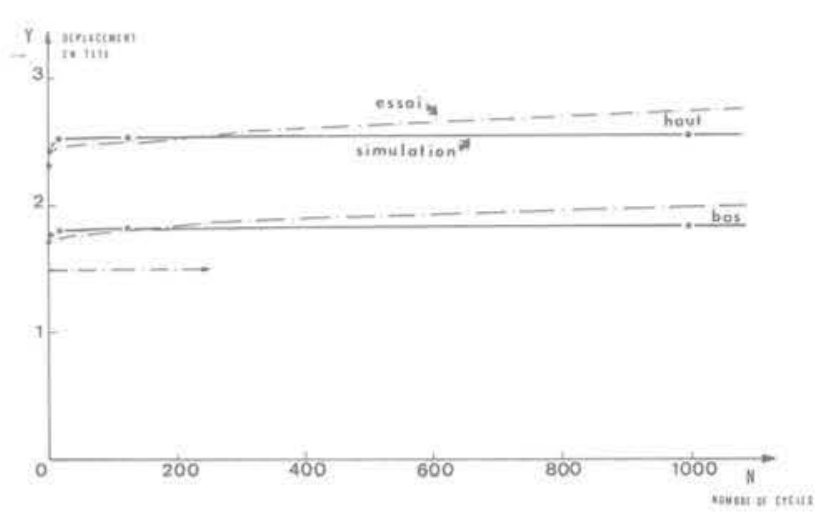

Fig. 13. - Plancoet essai cyclique C 12 Déplacement en tête haut et bas de cycles. 
lement et par simulation numérique (cf. fig. 14). Cet écart est important au cours du $1^{\text {er }}$ cycle $(-40 \%$ à + $60 \%$ ) mais s'amenuise au cours des cycles (-20\% à $+40 \%$ ) ce qui tendrait à prouver que les effets propres au cyclage sont pour leur part assez correctement simulès.

On rappelle que le «module de frottement» est la pente tangente de la courbe relation entre la contrainte de cisaillement locale et le déplacement relatif sol-pieu local.

4.3.2. Simulation de Pessai (arrachement cyclique) tempête $T 2-D$ -

Le programme de chargement simulé a été notablement simplifié par rapport au programme de chargement réel, et ramené à trois séries successives de cycles:

$$
\left\{\begin{array}{l}
1000 \text { cycles à } Q_{s t} \pm 50 \% \\
200 \text { cycles à } Q_{s t} \pm 75 \% \\
200 \text { cycles à } Q_{s t} \pm 100 \%
\end{array}\right.
$$

suivies d'un arrachement poursuivi jusqu'à rupture.

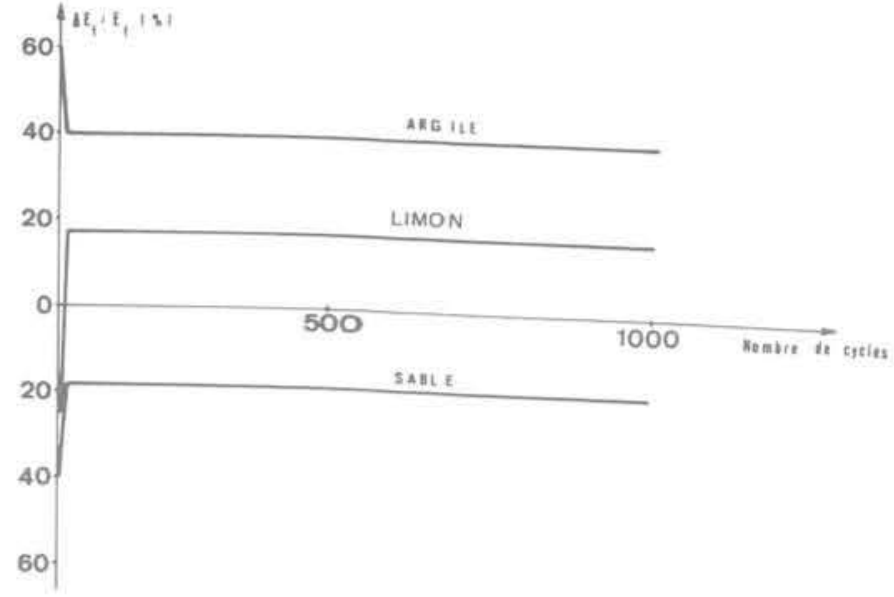

Fig. 14. - Ecart relatif $\frac{E_{f} \text { calculé }-E_{f} \text { mesuré }}{E_{f} \text { mesuré }}$ (simulation $C$ essai $C$ 12)

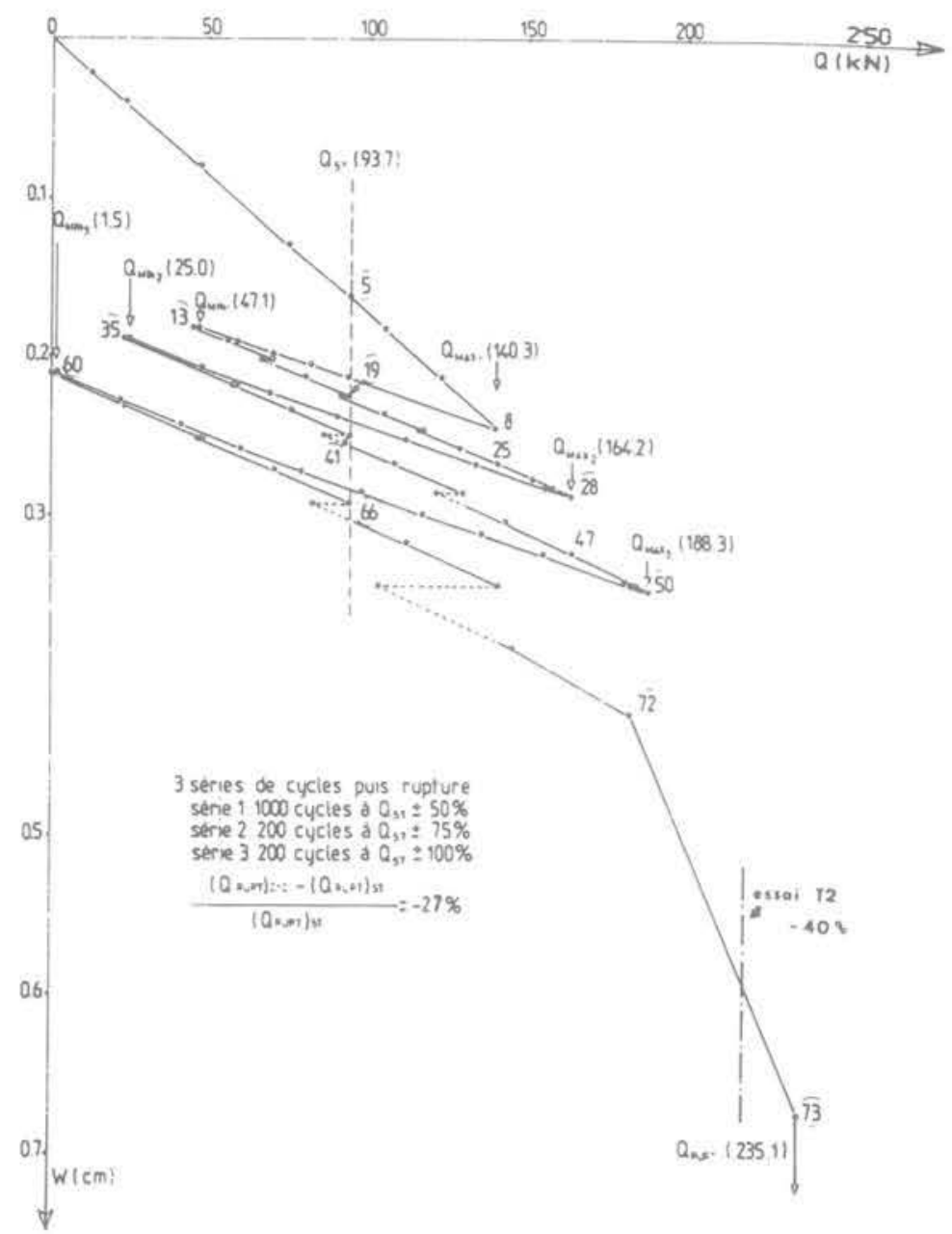

Fig. 15. - Simulation essai tempête T2. Pieu de Plancoet.

Courbe effort-arrachement. 


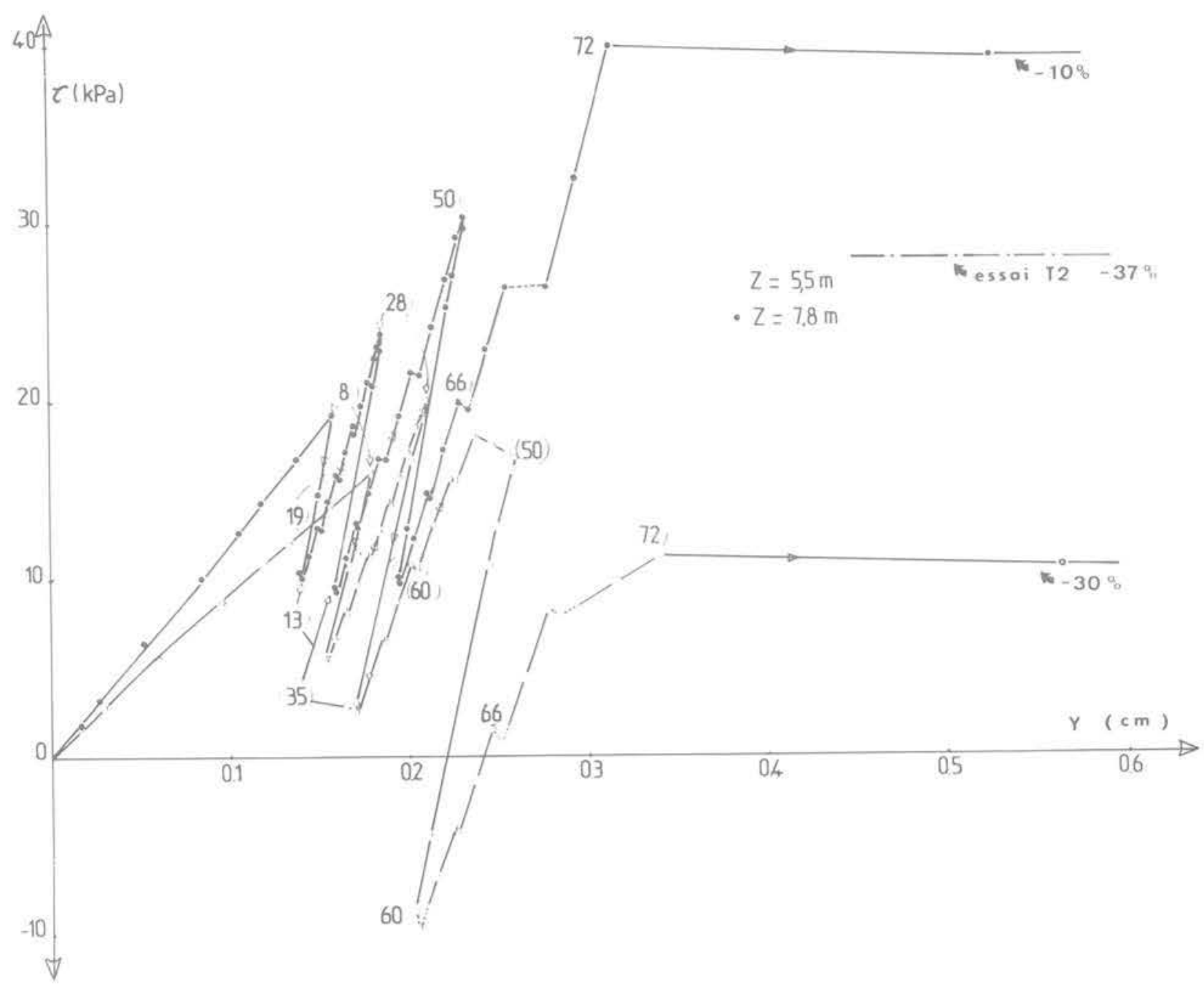

Fig. 16. - Plancoet mobilisation du frottement latéral.

Simulation essai tempête couche intermédiaire - sable lâche.

La charge moyenne $\mathrm{Q}_{\mathrm{st}}$ vaut $94 \mathrm{kN}$ (à comparer avec la charge de fluage). Cet ensemble constitue la simulation D. Cette simulation a été réalisée en 73 incréments, dont 12 (4 par série de cycles) de pseudo-fluage cyclique.

On constate sur la courbe effort arrachement simulée (cf. fig. 15, sur laquelle les $\mathrm{N}^{\circ}$ d'incréments figurent en paramètres), qu'il existe une tendance vers la rupture pour les cycles de grande amplitude (perceptible dès la seconde série de cycles, mais surtout lors de la troisième série).

Par ailleurs, le palier de force d'arrachement est réduit de $27 \%$ dans la simulation $D$, tandis qu'il est réduit expérimentalement de $40 \%$. Les parties tiretées de la courbe correspondent au pseudo-fluage cyclique.

La figure 16 montre l'évolution des contraintes de cisaillement dans la couche intermédiaire de sable lâche, au bout de cet ensemble de cycles. L'expérience donne une valeur moyenne de $-37 \%$, tandis que la simulation donne - $10 \%$ à $7,8 \mathrm{~m}$ de profondeur, et
- $30 \%$ à $5.5 \mathrm{~m}$ de profondeur; les numéros d'incréments sont portés en paramètres, comme sur la figure précédente. On peut noter que dans cette simulation la tendance était, malgré cette diminution pour des grands cycles, à l'augmentation des contraintes de cisaillement pour des petits cycles (cf. incrément $\left.n^{\circ} 19\right)$ conformément aux résultats de l'essai C 21 et de la simulation C.

Les déplacements en tête de haut et bas de cycles sont représentés à la figure 17 . Comme précédemment, le déplacement initial obtenu lors du premier cycle de chargement est mal rendu. Par contre, l'amplitude paraît correctement rendue. Mais ici, la variation du déplacement moyen au cours des cycles est également mal représentée. Ceci est un état de la méthode de calcul datant de près de deux années. Ce défaut a été corrigé en introduisant la notion de cycles équivalents dans toute sa rigueur: cette méthode de calcul, appliquée par la suite au pieu expérimental de Cran nous a donné satisfaction (1).

(1) Les résultats du calcul de Cran seront publiés ultérieurement par les auteurs. 


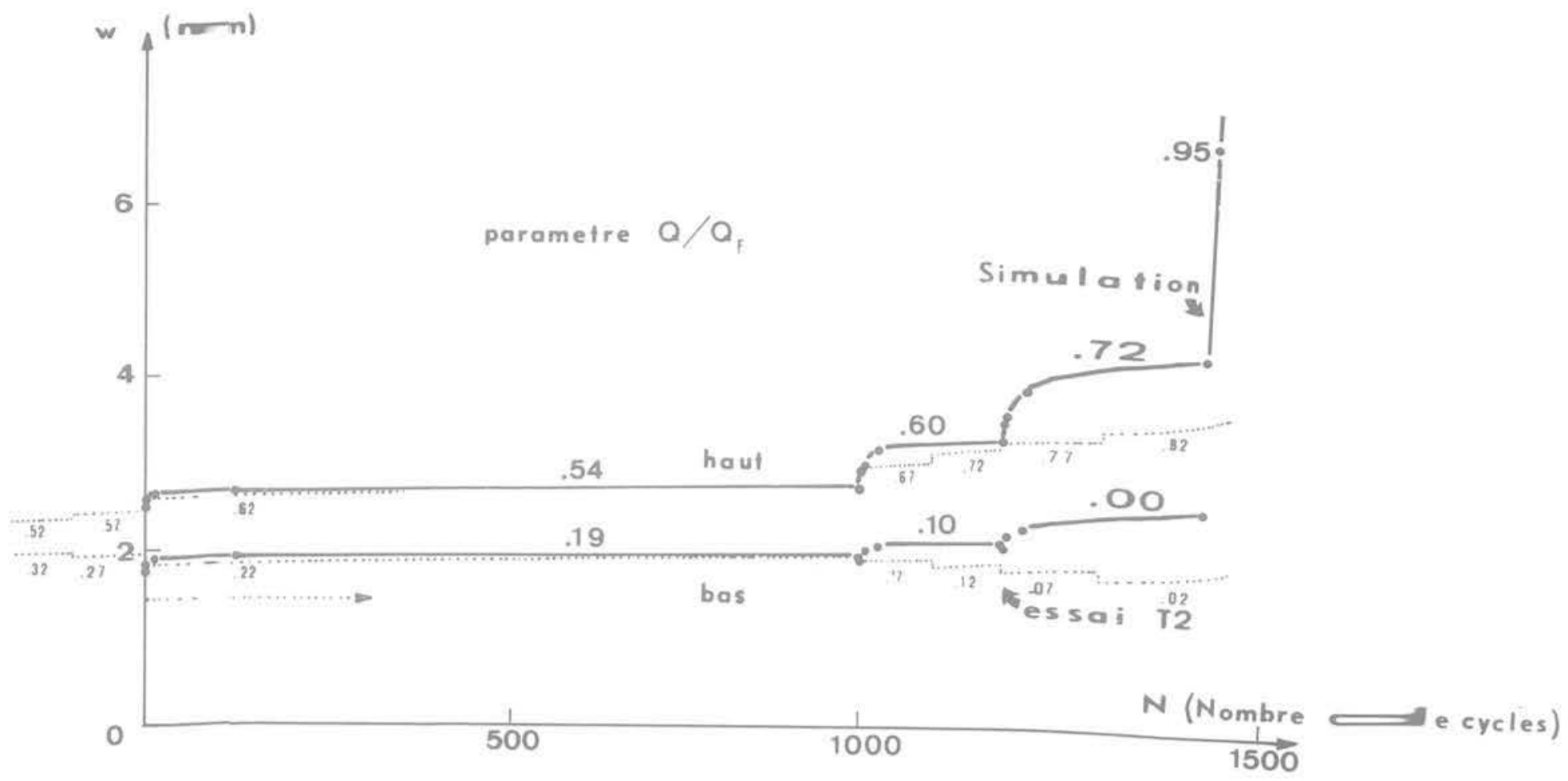

Fig. 17. - Plancoet simulation essai tempête.

Déplacements en tête haut et bas de cycles.

\section{CONCLU $\$ I O N$}

Le programme Cyclope de simulation numérique du comportement tes pieux sous chargement cyclique utilise la méth <de classique des éléments finis mais condense l'effe $t$ des cycles sous forme d'un pseudofluage cyclique. Cette approche a permis une simulation satisfaisant $€$ du comportement cyclique de pieux expérimentaux ivec un volume de calcul compatible avec les exigen tes courantes.

Cependant, ce type de simulation bute actuellement sur la schématis ation de l'état initial du sol, notamment en ce qui concerne les effets de la mise en place par battage.

L'intensification des travaux pétroliers en mer et la complexité croi ssante des structures mises en place posent plus que jamais le problème de la tenue et de l'optimisation d es fondations sur pieux de grandes tailles $(1<\varnothing<2 \mathrm{~m}, \mathrm{~L} \geqslant 50 \mathrm{~m})$ soumis à des sollicitations cyc liques axiales de forte amplitude.

De très gros efforts sont actuellement consentis par l'industrie pétrolière et parapétrolière pour améliorer les connaissances sur le comportement de ce type de fondation. Le programme de recherche conduit par l'I.F.P. s'est attaché à la fois à acquérir des données expérimentales originales sur des pieux très instrumentés et à promou voir le développement de méthodes de prévisions adaptées.
Parallèlement, l'Institut de mécanique de poursuit son effort tant sur le plan de la loi d'interface réaliste) physique grâce à une que sur le plan de la haute pression adaptée à la de calibratic ò à très longs pieux de l'Offshore. des très

\section{RÉFÉRENCES BIBLIOGRAPHIQUES}

1. ANDERSEN. - Behaviour of clay subj $\Longleftarrow$ cted to undrained cyclic loading, Proc. of the C—cted to Norvège, vol. 1, pp. 392-403.

2. BALAAM, N.P.; POULOS, H.G.; BOOKE - Finite element analysis of the effects tion on pile load settlement the effects of cal Engineering, vol. VI, no behaviour, Geœ techni-

BONIN J.P. 1975.

ZAMENHOF, L.C DELEUIL, G.; ZALESKIZAMENHOF, L.C. - Foundation analy_ - Sis of marine gravity structures submitted to $\mathrm{cycl}=\mathrm{C}$ of of U.S.A., 1976, paper 2475,

4. BOULON, M. ; CHAMBON, R. DARVE Loi rhéologique incrémentale pour les, $\quad \boldsymbol{F}$. applications par la méthode des éléments $\longrightarrow$ Is et Revue Française de Géotechnique éléments finis, tembre 1977. 